\title{
Determination of secondary ion mass spectrometry relative sensitivity factors for polar and non-polar $\mathrm{ZnO}$
}

\author{
Andreas Laufer, ${ }^{1, a)}$ Niklas Volbers, ${ }^{1}$ Sebastian Eisermann, ${ }^{1}$ Kay Potzger, ${ }^{2}$ \\ Sebastian Geburt, ${ }^{3}$ Carsten Ronning, ${ }^{3}$ and Bruno K. Meyer ${ }^{1}$ \\ ${ }^{1}$ 1. Physikalisches Institut, Justus-Liebig-Universität Giessen, Heinrich-Buff-Ring 16, 35392 Giessen, Germany \\ ${ }^{2}$ Helmholtz-Zentrum Dresden-Rossendorf, Bautzner Landstraße 400, 01328 Dresden, Germany \\ ${ }^{3}$ Institut für Festkörperphysik, Universität Jena, Max-Wien-Platz 1, 07743 Jena, Germany
}

(Received 12 July 2011; accepted 7 October 2011; published online 15 November 2011)

\begin{abstract}
Zinc oxide $(\mathrm{ZnO})$ is regarded as a promising material for optoelectronic devices, due to its electronic properties. Solely, the difficulty in obtaining p-type $\mathrm{ZnO}$ impedes further progress. In this connection, the identification and quantification of impurities is a major demand. For quantitative information using secondary ion mass spectrometry (SIMS), so-called relative sensitivity factors (RSF) are mandatory. Such conversion factors did not yet exist for $\mathrm{ZnO}$. In this work, we present the determined RSF values for $\mathrm{ZnO}$ using primary (ion implanted) as well as secondary (bulk doped) standards. These RSFs have been applied to commercially available $\mathrm{ZnO}$ substrates of different surface termination (a-plane, $\mathrm{Zn}$-face, and O-face) to quantify the contained impurities. Although these $\mathrm{ZnO}$ substrates originate from the same single-crystal, we observe discrepancies in the impurity concentrations. These results cannot be attributed to surface termination dependent RSF values for ZnO. ㅇ 2011 American Institute of Physics. [doi:10.1063/1.3660417]
\end{abstract}

\section{INTRODUCTION}

Zinc oxide $(\mathrm{ZnO})$ is a semiconductor with a direct band gap of $3.37 \mathrm{eV} .{ }^{1}$ Furthermore, $\mathrm{ZnO}$ is relatively abundant, non-toxic, chemically stable, easy to prepare, and inexpensive. Therefore, $\mathrm{ZnO}$ is a promising material for optoelectronic applications such as light-emitting diodes (LEDs) and photovoltaics. $^{2-4}$ Unfortunately, the difficulty of synthesizing p-type $\mathrm{ZnO}$ impedes the achievement of high quality ZnO-based optoelectronic devices. The severe difficulty in obtaining p-type $\mathrm{ZnO}$ has been partially attributed to intrinsic defects and impurities that act as compensating donors. ${ }^{5}$ To avoid these effects, it is, therefore, necessary to identify and quantify the impurities in the material.

Secondary ion mass spectrometry (SIMS) is a powerful analysis technique to determine the composition of solids and thin films. All elements and isotopes in the periodic table can be detected with high sensitivity (ppm to ppb) using this method. The SIMS measurements only provide qualitative information, due to the fact that the relative secondary ion intensities do not directly reflect the relative concentrations of the elements contained in such materials. For quantification, therefore, so-called relative sensitivity factors (RSF) are required to convert the measured secondary ion intensities into atomic concentrations in the investigated matrix. The RSF is defined by

$$
\rho_{i}=\frac{I_{i}}{I_{m}} \frac{A_{m}}{A_{i}} R S F_{i m},
$$

where $i$ indicates an impurity isotope, $m$ indicates the matrix isotope chosen for reference, $\rho_{i}$ is the impurity atom density

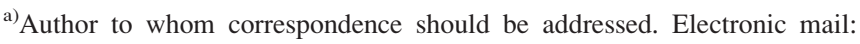
Andreas.Laufer@exp1.physik.uni-giessen.de.
}

(atoms $/ \mathrm{cm}^{3}$ ) in the matrix, $I$ is the secondary ion intensity (counts/s), and $A$ represents the corresponding isotope abundance. Unfortunately, the RSFs vary for each matrix and detected element. While there are RSF tables for $\mathrm{Si}$ and for compound semiconductors such as GaAs, InP or GaN, such a reference did not yet exist for $\mathrm{ZnO}$. In the present work, the RSFs for a number of technological important elements have been determined using ion implanted standards with known fluences (primary standard) and bulk doped samples with known impurity concentrations (secondary standard).

The determined RSFs have then been applied to commercially available $\mathrm{ZnO}$ substrates of different orientation and surface termination (a-plane, $\mathrm{Zn}$-face, and $\mathrm{O}$-face) to quantify the contained impurities. These results have been compared with published impurity concentrations of other groups (Sec. III B). Although our investigated $\mathrm{ZnO}$ substrates originate from the same single-crystal (further information in Sec. II), we observed differences in the impurity concentrations for a few elements depending on the orientation and the surface terminations. Therefore, we performed additional implantations $(\mathrm{H}, \mathrm{N}$, and $\mathrm{K})$ in $\mathrm{ZnO}$ substrates with different surface termination to test whether the RSFs depend on the surface termination of $\mathrm{ZnO}$.

\section{EXPERIMENTAL TECHNIQUES}

The primary standards for RSF determination have been produced via ion implantation (single implantation profile) of $\mathrm{ZnO}$ single-crystals (O-face) with the elements shown in Table I. The selected isotopes show no mass interferences with any zinc oxide related element or compound. For cobalt and manganese, the RSFs have been determined using doped zinc oxide thin films. For these samples, the concentrations have been determined using energy dispersive $\mathrm{x}$-ray spectroscopy (EDX). The maximum concentration for the 
TABLE I. List of implanted isotopes in $\mathrm{ZnO}$.

\begin{tabular}{|c|c|c|c|c|c|c|c|}
\hline \multirow{2}{*}{$\frac{\text { Matrix }}{\mathrm{ZnO}}$} & \multicolumn{7}{|c|}{ Implanted isotopes } \\
\hline & ${ }^{1} \mathrm{H}$ & ${ }^{7} \mathrm{Li}$ & ${ }^{14} \mathrm{~N}$ & ${ }^{23} \mathrm{Na}$ & ${ }^{27} \mathrm{Al}$ & ${ }^{31} \mathrm{P}$ & ${ }^{35} \mathrm{Cl}$ \\
\hline & ${ }^{57} \mathrm{Fe}$ & ${ }^{58} \mathrm{Ni}$ & ${ }^{69} \mathrm{Ga}$ & ${ }^{75}$ As & ${ }^{79} \mathrm{Br}$ & ${ }^{115} \mathrm{In}$ & ${ }^{127} \mathrm{I}$ \\
\hline
\end{tabular}

primary and secondary standards is in the range between $1 \times 10^{19}$ and $1 \times 10^{21} \mathrm{~cm}^{-3}$. To check the dependence of RSF values on surface termination (a-plane, $\mathrm{Zn}$-face, and O-face), hydrogen, nitrogen, and potassium have been implanted additionally as box-like profile in the respective $\mathrm{ZnO}$ singlecrystals. For potassium and the other two elements, the maximum implantation concentration is about $1 \times 10^{19} \mathrm{~cm}^{-3}$ and $1 \times 10^{21} \mathrm{~cm}^{-3}$, respectively. All ion implantations have been performed at room temperature using $7^{\circ}$ tilt angle from normal. The maximum implantation depth for a single implantation and a box-like implantation profile is typically in the range of $50-400 \mathrm{~nm}$ and $400-700 \mathrm{~nm}$, respectively. Post-implantation annealing processes have been performed depending on the implanted isotope and the implantation fluence.

The SIMS measurements have been performed using a Cameca MIQ256 instrument, equipped with an oxygen $\left(6 \mathrm{keV} \mathrm{O}_{2}^{+}\right.$primary ion beam energy) as well as a cesium primary ion source $\left(11 \mathrm{keV} \mathrm{Cs}{ }^{+}\right.$primary ion beam energy). For both ion beam species, the angle of incidence from normal is $45^{\circ}$. The primary ion current is adjusted to $40 \mathrm{nA}$ in each case. The scanned area is typically $250 \times 250 \mu \mathrm{m}^{2}$. To avoid crater edge effects, only secondary ions in a predefined area in the middle of the crater are counted (electronic gating). A quadrupole is used as mass analyzer and a channeltron as detector. The mass resolution $(\mathrm{m} / \Delta \mathrm{m})$ of our mass analyzer system is about 300 . The standards have been analyzed using oxygen primary ions for positive secondary ion mass spectrometry and cesium primary ions for negative secondary ion mass spectrometry.

The investigated $\mathrm{ZnO}$ substrates (a-plane, Zn-face, O-face) have been cut out of a $20 \times 20 \times 10 \mathrm{~mm}^{3}$ hydrothermally grown $\mathrm{ZnO}$ single crystal. The two polar $\mathrm{ZnO}$ substrates ( $\mathrm{Zn}$-face and $\mathrm{O}$-face) originate both from the $+c$ region of the $\mathrm{ZnO}$ single crystal. The a-plane $\mathrm{ZnO}$ substrate was cut out at the plane parallel to the $c$-axis ( $m$-region). The dimension of the three $\mathrm{ZnO}$ substrates is in each case about $10 \times 5 \times 0.5 \mathrm{~mm}^{3}$.

\section{RESULTS AND DISCUSSION}

\section{A. Relative sensitivity factors for $\mathrm{ZnO}$}

The determined RSFs for $\mathrm{ZnO}$ are presented in Table II. Higher RSF values indicate lower secondary ion yields and consequently higher detection limits. All stated RSFs are elemental RSFS, which means they refer to the sum of all isotopes of the impurity element as well as the matrix element (not specific isotopic RSFs). The matrix element for positive secondary ions (using $\mathrm{O}_{2}{ }^{+}$as primary ions) is $\mathrm{Zn}$. Due to the fact that $\mathrm{Zn}$ is not detectable as a negative ion, because of its electron affinity value of zero, the matrix element for negative secondary ions (using $\mathrm{Cs}^{+}$as primary ions) is $\mathrm{ZnO}$. The
TABLE II. Elemental RSFs for 16 elements in $\mathrm{ZnO}$ (matrix signal: $\mathrm{Zn}$ for positive secondary ions; $\mathrm{ZnO}$ for negative secondary ions).

\begin{tabular}{lcccc}
\hline \hline Elements & Ion & No. of Measurements & Primary ions & RSF (atoms $\left./ \mathrm{cm}^{3}\right)$ \\
\hline $\mathrm{H}$ & $\mathrm{H}^{+}$ & 1 & $\mathrm{O}_{2}{ }^{+}$ & $2.1 \times 10^{22}$ \\
$\mathrm{Li}$ & $\mathrm{Li}^{+}$ & 5 & $\mathrm{O}_{2}{ }^{+}$ & $2.2 \times 10^{19}$ \\
$\mathrm{~N}$ & $\mathrm{NO}^{-}$ & 4 & $\mathrm{Cs}^{+}$ & $3.6 \times 10^{23}$ \\
$\mathrm{Na}$ & $\mathrm{Na}^{+}$ & 3 & $\mathrm{O}_{2}{ }^{+}$ & $8.4 \times 10^{18}$ \\
$\mathrm{Al}$ & $\mathrm{Al}^{+}$ & 8 & $\mathrm{O}_{2}{ }^{+}$ & $1.0 \times 10^{20}$ \\
$\mathrm{P}$ & $\mathrm{PO}^{+}$ & 1 & $\mathrm{O}_{2}{ }^{+}$ & $2.9 \times 10^{22}$ \\
& $\mathrm{PO}^{-}$ & 4 & $\mathrm{Cs}^{+}$ & $8.6 \times 10^{21}$ \\
$\mathrm{Cl}$ & $\mathrm{Cl}^{+}$ & 2 & $\mathrm{O}_{2}{ }^{+}$ & $3.3 \times 10^{23}$ \\
& $\mathrm{Cl}^{-}$ & 3 & $\mathrm{Cs}^{+}$ & $1.3 \times 10^{21}$ \\
$\mathrm{Mn}$ & $\mathrm{Mn}^{+}$ & 1 & $\mathrm{O}_{2}{ }^{+}$ & $6.6 \times 10^{20}$ \\
$\mathrm{Fe}$ & $\mathrm{Fe}^{+}$ & 7 & $\mathrm{O}_{2}{ }^{+}$ & $1.2 \times 10^{21}$ \\
$\mathrm{Co}$ & $\mathrm{Co}^{+}$ & 2 & $\mathrm{O}_{2}{ }^{+}$ & $1.5 \times 10^{21}$ \\
$\mathrm{Ni}$ & $\mathrm{Ni}^{+}$ & 5 & $\mathrm{O}_{2}{ }^{+}$ & $4.3 \times 10^{21}$ \\
$\mathrm{Ga}$ & $\mathrm{Ga}^{+}$ & 3 & $\mathrm{O}_{2}{ }^{+}$ & $3.1 \times 10^{20}$ \\
$\mathrm{As}$ & $\mathrm{AsO}^{+}$ & 3 & $\mathrm{O}_{2}{ }^{+}$ & $2.3 \times 10^{22}$ \\
& $\mathrm{AsO}^{-}$ & 2 & $\mathrm{Cs}^{+}$ & $1.7 \times 10^{22}$ \\
$\mathrm{Br}$ & $\mathrm{Br}^{+}$ & 1 & $\mathrm{O}_{2}{ }^{+}$ & $6.7 \times 10^{23}$ \\
& $\mathrm{Br}^{-}$ & 2 & $\mathrm{Cs}^{+}$ & $1.1 \times 10^{21}$ \\
$\mathrm{In}$ & $\mathrm{In}^{+}$ & 2 & $\mathrm{O}_{2}{ }^{+}$ & $1.8 \times 10^{21}$ \\
$\mathrm{I}$ & $\mathrm{I}^{-}$ & 2 & $\mathrm{Cs}^{+}$ & $3.6 \times 10^{21}$ \\
\hline \hline
\end{tabular}

uncertainty in the RSF values is in the range of $\pm 30 \%-50 \%$ depending on signal-to-noise ratio and impurity content.

For further elements for which no standards have been prepared so far, RSF values have been interpolated and extrapolated using the systematic trends for positive SIMS RSFs described in detail by Wilson et al. ${ }^{6-8}$ Figure 1 shows all calculated, interpolated, and extrapolated positive SIMS RSFs for $\mathrm{ZnO}$ as a function of atomic number. The RSF values increase across each period or downward within a group of the periodic table. This is the same behavior which can be found for the ionization potentials. For the negative SIMS RSFs, no explicit systematic trends can be found. ${ }^{7}$ Therefore, no negative SIMS RSFs have been interpolated or extrapolated.

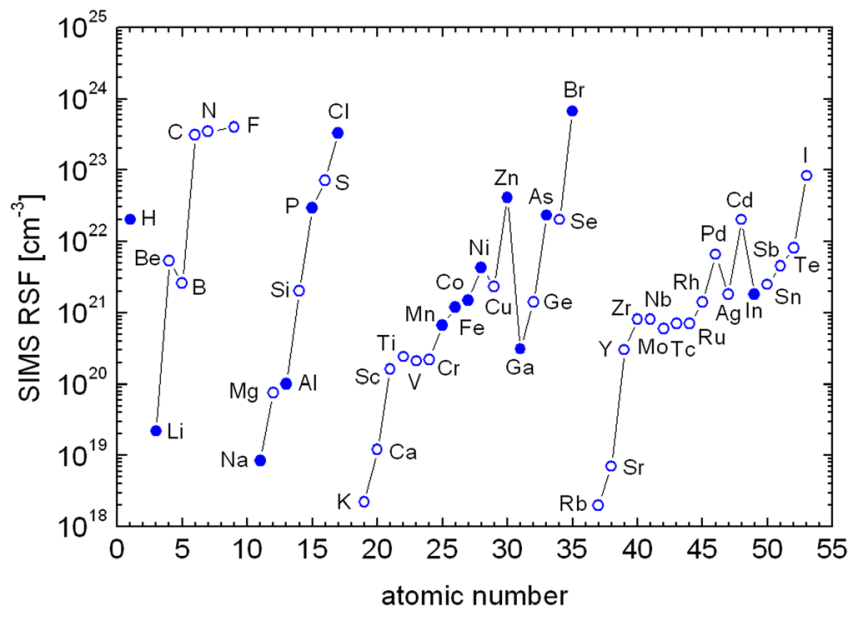

FIG. 1. (Color online) Positive SIMS RSFs as function of atomic number (closed circle-calculated via standard; open circle-inter- or extrapolated value). 


\section{B. Impurities in hydrothermally grown $\mathrm{ZnO}$}

The determined RSFs for $\mathrm{ZnO}$ have been used to calculate the impurity concentrations of hydrothermally grown $\mathrm{ZnO}$ substrates. We investigated polar (Zn-face and O-face) and nonpolar (a-plane) $\mathrm{ZnO}$ crystals which originate from the same single-crystal. In Figure 2, the impurity concentrations for the samples with different surface termination are shown. The majority of impurity concentrations is below $1 \times 10^{18}$ atoms $/ \mathrm{cm}^{3}$. In spite of using a mixture of $\mathrm{KOH}$ and $\mathrm{LiOH}$ aqueous solution in the hydrothermal growth process, the detected potassium and lithium concentrations are relatively moderate (about $1 \times 10^{17}$ to $1 \times 10^{18}$ atoms $/ \mathrm{cm}^{3}$ ). Only for the elements $\mathrm{H}, \mathrm{C}, \mathrm{Si}, \mathrm{N}$, and $\mathrm{Cl}$ did we detect impurity concentrations above $1 \times 10^{18}$ atoms/ $\mathrm{cm}^{3}$. The hydrogen and nitrogen impurity concentrations slightly exceed the respective detection limit. Further potential impurities, e.g. Fe, are below the according detection limit.

In Table III, we compare our results with the impurity concentrations in hydrothermally grown $\mathrm{ZnO}$ single crystals published by other groups. ${ }^{9-12}$ Only the concentrations for lithium, potassium, aluminum, and iron have been measured by all groups. The large discrepancy of the values (Table III) may be attributed in parts to the use of different units (wt. ppm and at. ppm). The impurity concentrations from Sekiguchi $e t$ al. ${ }^{12}$ as well as our values are given in at. ppm. The concentrations reported by the other three groups are most likely given in wt. ppm. If this is the case, these values will be a bit larger in units of at. ppm according to the mass (e.g., $\sim \times 10$ for $\mathrm{Li}, \sim \times 3$ for $\mathrm{Al}$ ). The lowest impurity concentrations have been found by Ehrentraut et al. ${ }^{9}$ and Ohshima et al. ${ }^{11}$ in $\mathrm{ZnO}$ substrates originating from the $+c$ region. Our lowest concentrations are comparable with these results. Only for iron we have detected lower concentrations $(<0.5$ at. ppm). On the other hand, the impurity concentrations reported by Sekiguchi et al. ${ }^{12}$ are about one order of magnitude higher than our measured concentrations.

\section{RSF dependence on surface termination}

For most of the impurities (Figure 2), the calculated concentrations for the different orientations vary only according

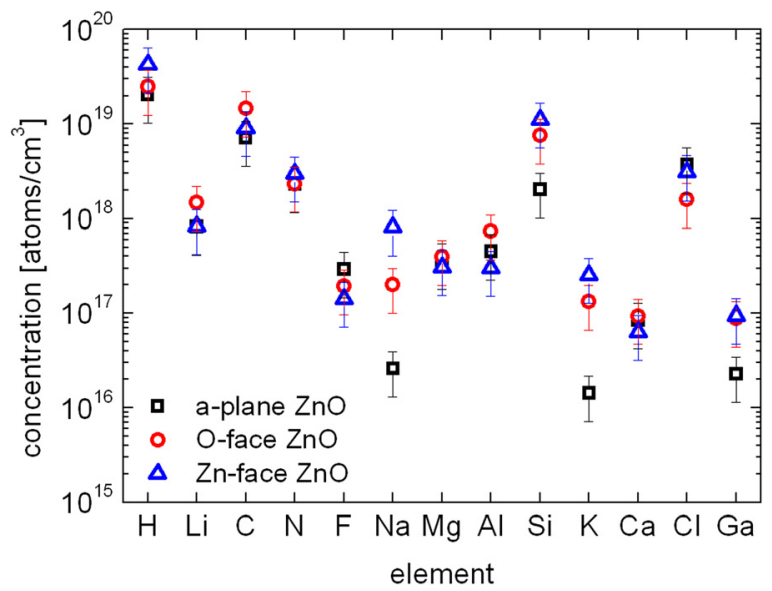

FIG. 2. (Color online) Impurity concentrations for polar and non-polar $\mathrm{ZnO}$ substrates ( $\mathrm{Zn}$-face, O-face, and a-plane) originating from the same singlecrystal.
TABLE III. A comparison of our results with the impurity concentrations published by other groups. Large discrepancy of the values may be attributed to the use of different units (wt. ppm and at. ppm). Our results are given in at. ppm.

\begin{tabular}{lccccc}
\hline \hline \multicolumn{5}{c}{ Concentration (ppm) } \\
\cline { 2 - 6 } & $\begin{array}{c}\text { Ehrentraut } \\
\text { Elements }\end{array}$ & $\begin{array}{c}\text { Mass } \\
\text { et } \text { (Ref. 9) }\end{array}$ & $\begin{array}{c}\text { Ohshima } \\
\text { et. (Ref. 10) }\end{array}$ & $\begin{array}{c}\text { Sekiguchi } \\
\text { et. (Ref. 11) }\end{array}$ & $\begin{array}{c}\text { Our } \\
\text { et. (Ref. 12) } \\
\text { work }\end{array}$ \\
\hline $\mathrm{Li}$ & $0.5-12$ & $1-10$ & $0.5-12$ & $30-180$ & 13 \\
$\mathrm{~K}$ & $<0.3$ & $<2$ & $0.04-0.2$ & $10-150$ & $0.1-4$ \\
$\mathrm{Al}$ & $0.1-8$ & $1-10$ & $0.1-2$ & $<30$ & $4-10$ \\
$\mathrm{Fe}$ & $0.5-11$ & $1-10$ & $0.5-11$ & $4-9$ & $<0.5$ \\
\hline \hline
\end{tabular}

to the accuracy of measurement. However, for a few elements $(\mathrm{Na}, \mathrm{Si}, \mathrm{K}$, and $\mathrm{Ga})$, we observed discrepancies in impurity concentration which cannot be explained by the uncertainty of measurement (30\%-50\%). The impurity concentrations in a-plane $\mathrm{ZnO}$ seem to be in each case the lowest compared with $\mathrm{Zn}$ - and $\mathrm{O}$-face $\mathrm{ZnO}$. For sodium and potassium, the RSF values differ by 1-2 orders of magnitude for the investigated orientations.

We performed additional SIMS measurements at six different points (see Figure 3) of the a-plane $\mathrm{ZnO}$ to check if there is a gradient in impurity concentration along the surface. Due to the dimension of the a-plane $\mathrm{ZnO}$ substrate, a large area parallel to the $c$-axis of the $\mathrm{ZnO}$ ingot is covered. Figure 3 illustrates the impurity concentrations, which differ in our results (Figure 2) or showed strong position dependence referred to the $c$-axis as reported by Ehrentraut et al. ${ }^{9}$ It is immediately obvious that there is no gradient in impurity concentration observable along the surface.

These facts raise the question if the RSFs may be dependent on the surface termination. To check this issue, therefore, we chose potassium due to its low impurity level and the distinct discrepancies in impurity concentration. Potassium was implanted in polar and nonpolar zinc oxide substrates as a boxlike profile (maximum implantation concentration $1 \times 10^{19}$ atoms $/ \mathrm{cm}^{3}$ ). In addition, we selected the elements $\mathrm{H}$ and $\mathrm{N}$ to

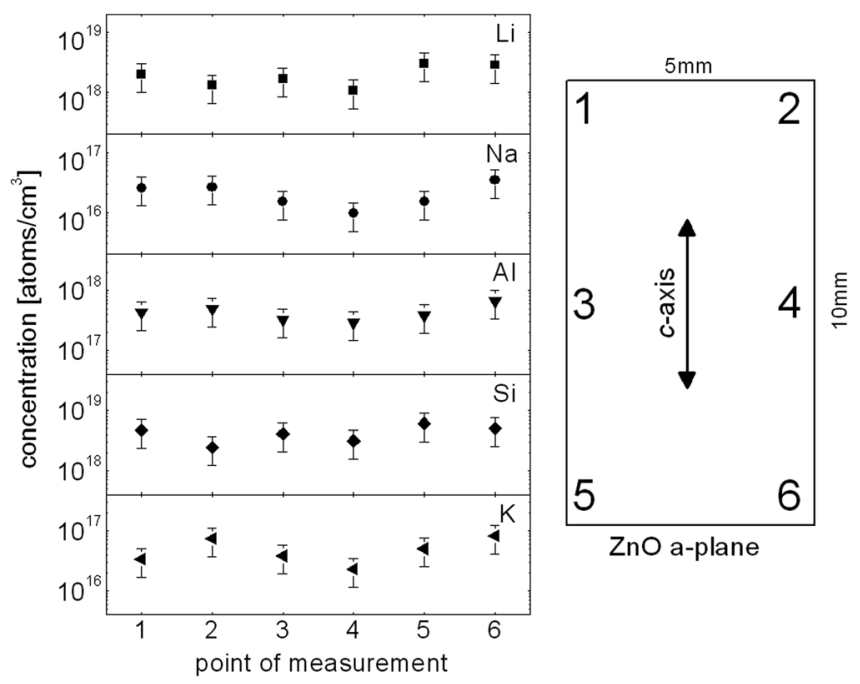

FIG. 3. Concentration of $\mathrm{Li}, \mathrm{Na}, \mathrm{Al}, \mathrm{Si}$, and $\mathrm{K}$ in a-plane $\mathrm{ZnO}$ depending on the point of measurement. 
determine the effect of surface termination on RSF value, due to the fact that the observed impurity concentrations for hydrogen and nitrogen lie slightly above their respective detection limit. In this case, a variation in impurity concentration regarding the surface termination may be concealed. Hydrogen and nitrogen have been implanted in polar zinc oxide substrates as box-like profiles. The maximum implantation concentration is $1 \times 10^{21}$ atoms $/ \mathrm{cm}^{3}$ due to the relatively high impurity level for these two elements.

For these three additional elements, we initially determined the RSFs for the respective surface terminations of the as-implanted $\mathrm{ZnO}$ substrates. We observed evidence in the depth profiles only for the potassium implantations that the crystal structure might be slightly damaged by the implantation process. Figure 4(a) shows the depth profile of the $\mathrm{K}$-implanted a-plane $\mathrm{ZnO}$ sample (as-implanted) representative for all orientations. Although a box-like implantation profile has been implanted, an increase in all secondary ion signals (K, Zn, and $\mathrm{O}$ ) with increasing depth can be observed. This variation in secondary ion intensities indicates a modification of the $\mathrm{ZnO}$ matrix caused by the ion implantation. The highest modification emerges near the surface as expected for ion implantion. ${ }^{13}$ To exclude any influences on RSF value due to the slight implantation damages, the implanted samples have been annealed at $700{ }^{\circ} \mathrm{C}$ for $60 \mathrm{~s}$ under nitrogen atmosphere. In Figure 4(b), the depth profile of the annealed $\mathrm{K}$-implanted a-plane $\mathrm{ZnO}$ sample is presented. In contrast to the as-implanted sample (Figure 4(a)), the potassium and the matrix signals are constant in the plateau region of the implantation profile. All calculated RSFs for the three additional elements, the different orientations,
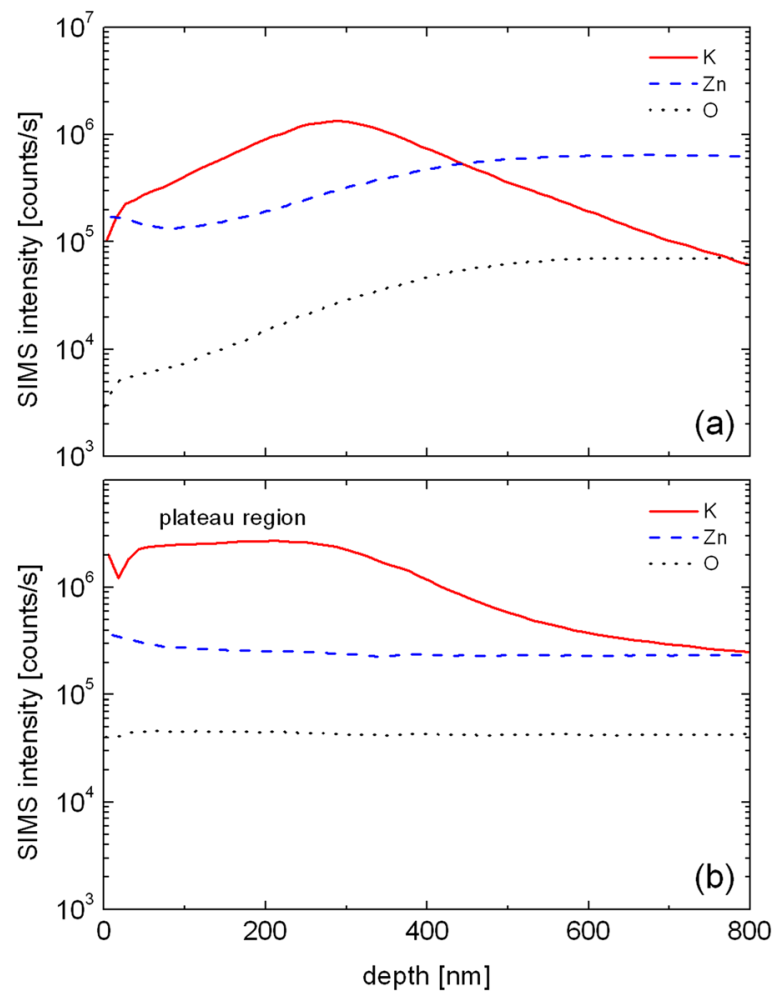

FIG. 4. (Color online) A comparison between the depth profiles of the as-implanted (a) and the annealed (b) a-plane $\mathrm{ZnO}: \mathrm{K}$ substrates.
TABLE IV. A comparison of RSF values for different surface terminations for as-implanted and annealed $\mathrm{ZnO}$ substrates. The uncertainty in RSF values is about $30 \%$.

\begin{tabular}{|c|c|c|c|c|c|c|}
\hline \multirow[b]{3}{*}{ Elements } & \multicolumn{6}{|c|}{$\operatorname{RSF}\left(\mathrm{cm}^{-3}\right)$} \\
\hline & \multicolumn{3}{|c|}{ As-implanted } & \multicolumn{3}{|c|}{ Annealed $\left(700^{\circ} \mathrm{C}, 60 \mathrm{~s}, \mathrm{~N}_{2}\right)$} \\
\hline & O-face & Zn-face & a-plane & O-face & Zn-face & a-plane \\
\hline $\mathrm{K}$ & $2.8 \times 10^{18}$ & $2.6 \times 10^{18}$ & $2.5 \times 10^{18}$ & $2.1 \times 10^{18}$ & $2.4 \times 10^{18}$ & $2.2 \times 10^{18}$ \\
\hline $\mathrm{H}$ & $2.2 \times 10^{22}$ & $2.9 \times 10^{22}$ & - & - & - & - \\
\hline $\mathrm{N}$ & $2.9 \times 10^{23}$ & $3.4 \times 10^{23}$ & - & $3.1 \times 10^{23}$ & $3.5 \times 10^{23}$ & - \\
\hline
\end{tabular}

and the treatment are listed and compared in Table IV. The uncertainty of all RSFs stated in Table IV is about $30 \%$. Although serious differences are observable between the depth profiles for the as-implanted and the annealed K-implanted $\mathrm{ZnO}$ samples (Figure 4), the determined RSF values are identical (Table IV). For nitrogen, the RSFs for the different orientations are identical among each other and between the untreated and annealed samples as well. Unfortunately, it was not possible to investigate the hydrogen implanted samples after the annealing step due to a complete outward diffusion of hydrogen. But for the asimplanted samples ( $\mathrm{Zn}$ - and O-face), the RSF values vary only according to the accuracy of measurement. Therefore, the secondary ion yields are not dependent on the surface termination.

Consequently, the differences in the detected impurity concentrations may originate from a crystal orientation dependent impurity incorporation during a fabrication step. Such a dependence of impurity incorporation also has been observed by Ehrentraut et al. ${ }^{9}$ and Ohshima et al. ${ }^{11}$ Several sources are imaginable. First, impurities in the aqueous solution of the hydrothermal growth process may be incorporated differently according to the surface termination of the $\mathrm{ZnO}$ crystal. Second, different impurity concentrations for the respective surface orientations of the seed crystal could be the source for the discrepancies in the impurity concentrations. Third, the different impurity incorporations may take place in a post-growth annealing process. Further investigation is needed to clarify the origin.

\section{CONCLUSION}

The determined RSFs allow the quantification of impurities in $\mathrm{ZnO}$. Furthermore, we show that the RSFs are independent of crystal orientation. Hence, now it is possible to quantify (depth-resolved) elements which are considered as acceptors (e.g., nitrogen) and elements which tend to act as compensating donors (e.g., interstitial alkali metals) in $\mathrm{ZnO}$. This knowledge will be mandatory to control the synthesis of $p$-type $\mathrm{ZnO}$ concerning the impurity perspective. ${ }^{14}$ To verify the origin of the differing impurity concentration depending on the orientation and surface termination, further investigation is needed.

${ }^{1}$ C. F. Klingshirn, B. K. Meyer, A. Waag, A. Hoffmann, and J. Geurts, Zinc Oxide-From Fundamental Properties Towards Novel Applications (Springer, Berlin, 2010).

${ }^{2}$ D. C. Look, Mater. Sci. Eng., B 80, 383 (2001). 
${ }^{3}$ D. C. Look, J. Electron. Mater. 35, 1299 (2006).

${ }^{4} \ddot{U}$. Özgür, Ya. I. Alivov, C. Lin, A. Teke, M. A. Reshchikov, S. Dogan, V. Avrutin, S.-J. Cho, and H. Morkoc, J. Appl. Phys. 98, 041301 (2005).

${ }^{5}$ K. G. Saw, K. Ibrahim, Y. T. Lim, and M. K. Chai, Thin Solid Films 515, 2879 (2007).

${ }^{6}$ R. G. Wilson, F. A. Stevie, and C. W. Magee, Secondary Ion Mass Spectrometry (Wiley, New York, 1989).

${ }^{7}$ R. G. Wilson and S. W. Novak, J. Appl. Phys. 69, 466 (1991).

${ }^{8}$ R. G. Wilson, J. Appl. Phys. 63, 5121 (1988).
${ }^{9}$ D. Ehrentraut, H. Sato, Y. Kagamitani, H. Sato, A. Yoshikawa, and T. Fukuda, Prog. Cryst. Growth Charact. 52, 280 (2006).

${ }^{10} \mathrm{~J}$. Mass, M. Avella, J. Jiménez, M. Callahan, E. Grant, K. Rakes, D. Bliss, and B. Wang, Mater. Res. Soc. Symp. Proc. 878E, Y1.7.1. (2005).

${ }^{11}$ E. Ohshima, H. Ogino, I. Niikura, K. Maeda, M. Sato, M. Ito, and T. Fukuda, J. Cryst. Growth 260, 166 (2004).

${ }^{12}$ T. Sekiguchi, S. Miyashita, K. Obara, T. Shishido, and N. Sakagami, J. Cryst. Growth 214/215, 72 (2000).

${ }^{13}$ H. Ryssel and I. Ruge, Ionenimplantation (B. G. Teubner, Stuttgart, 1978).

${ }^{14}$ C. H. Park, S. B. Zhang, and S.-H. Wei, Phys. Rev. B 66, 073202 (2002). 